Gefässchirurgie 2021 $26: 366-371$ https://doi.org/10.1007/s00772-021-00806-9 Angenommen: 16. Juli 2021

Online publiziert: 18. August 2021 Springer Nature 2021
๑) Springer Medizin Verlag GmbH, ein Teil von

\section{Innovativ: geriatrische Konzepte für die Gefäßmedizin und Gefäßchirurgie}

\author{
Björn Maassen · Konstantinos Chondros · Leo Cornelius Bollheimer \\ Medizinische Klinik VI (Altersmedizin), Uniklinik RWTH Aachen, Aachen, Deutschland
}

\section{Zusammenfassung}

\section{In diesem Beitrag}

- "Der Mensch ist so alt wie seine Gefäße" ein Trugschluss?

- Synergien zwischen Gefäßmedizin und Geriatrie

Multimorbidität • Polypharmazie • Frailty • Delir - Fallstricke für und Chancen durch einen besseren Synergismus

- Gefäßmedizinische Entitäten im Kontext der ferneren Lebenserwartung

Entwicklungen und Fortschritte in Gefäßchirurgie und Geriatrie verlaufen bis dato weitgehend unabhängig voneinander; auch im klinischen Alltag der Krankenversorgung gibt es bislang - anders als bei der Alterstraumatologie - kaum Überschneidungen und Synergismen. Dabei wären interdisziplinär umgesetzte geriatrische Konzepte bei der individualisierten Indikationsstellung, Therapiewahl und Prognoseabschätzung in der Gefäßchirurgie sicherlich hilfreich, v.a. bei hochaltrigen Patienten (85+ Jahre). Geriatrische Begrifflichkeiten wie biologisches Alter und Funktionalität sind dabei kein alleiniges Spiegelbild des Gefäßstatus, sondern schließen neben weiteren organspezifischen Komponenten (z. B. Immunologie, muskuloskelettales System etc.) v. a. auch die psychisch-neurokognitive Domäne und

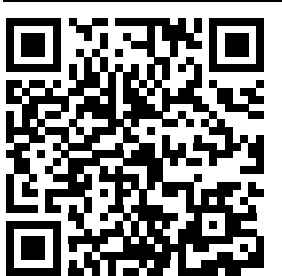

QR-Code scannen \& Beitrag online lesen sozialmedizinische Kontextfaktoren ein. Eine Beschränkung auf das kalendarische Alter, den Gefäßstatus oder auch schlagwortartige geriatrische Surrogatparameter wie Frailty wird dabei der gesundheitlichen Charakterisierung von alten Menschen nicht gerecht. Im vorliegenden Artikel werden die Gedankengänge an der Schnittstelle zwischen Gefäßchirurgie und Geriatrie am Beispiel des Bauchaortenaneurysmas (BAA) und der peripheren arteriellen Verschlusskrankheit (pAVK) vertieft.

\title{
Schlüsselwörter
}

Bauchaortenaneurysma · Periphere arterielle Verschlusskrankheit · Multimorbidität . Polypharmazie · Frailty

\section{„Der Mensch ist so alt wie seine Gefäße" - ein Trugschluss?}

Die leider noch weitgehende Parallelexistenz von Gefäßmedizin auf der einen Seite und Geriatrie auf der anderen wird in Deutschland durch das gleichlautende Akronym für beide Fachgesellschaften - DGG - konterkariert: Entsprechend findet sich der Geriater ${ }^{1}$ beim suchportalgesteuerten Internetaufruf bisweilen irrtümlicherweise bei der Deutschen Gesellschaft für Gefäßchirurgie und Gefäßmedizin (https://www.gefaesschirurgie.de) und vice versa der Gefäßchirurg bei der Deut-

\footnotetext{
1 Aus Gründen der besseren Lesbarkeit wird auf die gleichzeitige Verwendung der Sprachformen männlich, weiblich und divers verzichtet. Sämtliche Personenbezeichnungen gelten gleichermaßen für alle Geschlechter.
}

schen Gesellschaft für Geriatrie (https:// www.dggeriatrie.de) wieder.

Während in anderen chirurgischen Bereichen - allen voran der Alterstraumatologie („orthogeriatrics") - das interdisziplinäre Crossover zu nachgewiesenermaßen höheren Behandlungserfolgen geführt und sich im Krankenhausalltag etablierthat [1], wird geriatrische Gefäßmedizin als berechtigte(!) Wunschvorstellung bislang nur in Fachkongressen theoretisiert und mit Ausnahme des Trendbegriffs der Gebrechlichkeit („frailty", s. unten) selbst im Schrifttum kaum adressiert [2-4].

Die fachliche Distanz zwischen Gefäßmedizin und Geriatrie ist verwunderlich, betrifft doch eine wesentliche Kernkompetenz des Gefäßmediziners die Atherosklerose und damit gerade die Menschen, welche typischerweise über lange Lebensjahre hinweg eine arteriosklerotische Beein- 
trächtigung von Körperfunktion und Körperstruktur akkumuliert haben [5].

Mit dem fälschlicherweise Rudolf Virchow (Zitat stammt eher von dem Pathologen Robert Rössle, 1876-1956) zugeschriebenen Aphorismus "Der Mensch ist so alt wie seine Gefäße" läuft die Gefäßmedizin Gefahr, sich überzogenen Ansprüchen im Sinne von antiaging auszusetzen. Vielmehr muss in der geriatrischen Gefäßmedizin das Motto „adding life to years" [6] mit funktionsbegründeten Therapiemaßnahmen im Vordergrund stehen und das jeweilige Alter im Sinne eines pro-aging als unabänderliche Größe akzeptiert werden. Generell gilt nämlich, dass mit zunehmendem Lebensalter präventive und systemische Therapieansätze zunehmend in den Hintergrund treten und stattdessen symptomatische bzw. unmittelbar funktionsverbessernde Maßnahmen den Behandlungsplan bestimmen.

Der Umkehrschluss zum oben erwähnten Pseudo-Virchow-Zitat, im Sinne dass der Mensch so jung wäre wie seine Gefäße, erscheint im Hinblick auf realistische Behandlungsziele einer modernen Gefäßmedizin noch irreführender: Biologisch junge Gefäße sind weder eine pars pro toto noch ein Garant für eine sog. Diatrigerie, d.h. einem verlangsamten biologischen Altern in Relation zum kalendarischen Alter. Zu viele Faktoren müssen letztendlich für ein umfassendes biologisches Jung-Bleiben aufeinandertreffen, was letztendlich auch die große interindividuelle Streubreite der funktionalen Gesundheit im Alter erklärt. Exemplarische Prägnanztypen wären hier die rüstige und selbstständig wohnende 90jährige Seniorin ohne jeglichen Medikamentenbedarf auf der einen Seite und der erst 70-jährige, unter Betreuung stehende Pflegeheimbewohner mit Polypharmazie (gängige Definition $\geq 5$ Medikamente [7]) und Multimorbidität (gängige Definition $\geq 2$ aktivitätsmindernde Erkrankungen [8]).

Diese Denkweise findet sich auch in der deutschsprachigen Konsensusdefinition zum geriatrischen Patienten wieder, nach der eine Person zwischen 70 und 79 Jahren nur bei Vorliegen einer geriatrietypischen Multimorbidität (d.h. $\geq 2$ Erkrankungen mit sozialmedizinisch aktivi- tätsmindernden Auswirkungen) als geriatrisch gilt. Ab einem Alter von 80 Jahren kann aufgrund der alterstypisch erhöhten Vulnerabilität per definitionem zwar generell von einem geriatrischen Phänotyp ausgegangen werden, doch gibt es eben auch hier Ausnahmen (s. oben Prägnanztypen; [9]). Die interindividuelle, vom kalendarischen Alter unabhängige Heterogenität bedingt das ebenso unabhängige biologische Alter und ist Resultat einer Interaktion von multiplen Beeinträchtigungen der Körperfunktion und Körperstruktur bzw. umgekehrt ein Ausdruck der bis dahin erhaltenen oder verlorengegangenen Ressourcen. Die Einflussfaktoren des biologischen Alters betreffen dabei eben nicht nur den Fachbereich der Gefäßchirurgie, sondern in gleichem Maße und unabhängig davon multiple andere medizinische Formenkreise, wie beispielsweise neurodegenerativen Erkrankungen etc.

\section{Synergien zwischen Gefäßmedizin und Geriatrie}

\section{Multimorbidität}

Multimorbidität stellt einen besonderen Behandlungsschwerpunkt der Geriatrie dar. Die alleinige Betrachtung der im beispielsweise akuten Krankheitsfall führenden Haupterkrankung führt meist nur zu einer unzureichenden Wiederherstellung des prämorbiden Status, da häufig parallel andere chronische Erkrankungen exazerbieren können. Um die Auswirkungen der Multimorbidität auf die Alltagskompetenzen des alten Menschen zu messen, nutzt die Altersmedizin das Tool des umfassenden geriatrischen Assessments (,comprehensive geriatric assessment ${ }^{\prime \prime}$ ) als multiprofessionelle Teamarbeit [10]. Durch das Assessment können individuelle, therapierelevante Defizite aus Bereichen wie körperliche Funktionsfähigkeit, geistige Fähigkeiten, Mangel- und Fehlernährung, Aktivitäten des täglichen Lebens und der sozialen Umstände identifiziert und in der interdisziplinären Betreuung durch Ärzte, Pflege-, Physio- und Ergotherapeuten, Psychologen, Logopäden, Diabetesund Ernährungsteam und Sozialdienst adressiert werden [11, 12].

\section{Polypharmazie}

Aus der Multimorbidität, welche im Alter und gerade bei gefäßmedizinisch erkrankten Patienten sehr häufig das kardiovaskuläre System betrifft, resultiert in der Regel die ebenfalls schon erwähnte Polypharmazie. Mit dem korrekten Umgang dieser - insbesondere beim alten Menschen häufig aus ganz unterschiedlichen Fachrichtungen stammenden - Arzneimittel kann man sich leicht überfordert fühlen, insbesondere wenn die Verordnungen nicht den genuinen Fachbereich betreffen.

So sollte die akut notwendige Verschreibung (z. B. eines Analgetikums) beim alten Menschen immer auf eine Eignung in dieser Altersgruppe geprüft werden. Hilfestellung bieten hierbei sogenannte PIM-Listen (potenziell inadäquate Medikation im Alter) wie beispielsweise die für den deutschsprachigen Raum geeignete FORTA- („Fit fOR The Aged"; [13]) oder die PRISCUS-Liste [14], die jedoch nur als Orientierungshilfe dienen und keinesfalls eine eigenverantwortliche kritische Prüfung vor dem Hintergrund der Gesamtsituation der Patienten und deren Vorerkrankungen ersetzt. Insbesondere die FORTA-Liste ist mittlerweile schon mehr als 10 Jahre alt und berücksichtigt daher nicht in der Zwischenzeit erschienene Rote-Hand-Briefe (z. B. zu Domperidon oder zu Diclofenac). Zudem sollte stets auch ein Abgleich mit den in der Fachinformation empfohlenen Dosisanpassungen erfolgen. Die Polypharmazie im Alter mit allen daraus resultierenden Problemen wird in der Altersmedizin gezielt mit z.B. Prüfungen auf Arzneimittel ohne Indikation, Indikationen ohne Arzneimitteltherapie, Kontraindikationen, notwendige Dosisanpassungen, Interaktionen sowie unerwünschte Arzneimittelwirkungen und daraus resultierende Verschreibungskaskaden gezielt adressiert. Vor dem Hintergrund dieser speziellen gerontopharmakologischen Kompetenz gehört es zu den Kernaufgaben der Geriatrie, insbesondere hinsichtlich der Arzneimitteltherapie im perioperativen Management Hilfestellungen zu geben.

\section{Frailty}

Für die Gefäßmedizin und Gefäßchirurgie gibt es diverse neuere Studien, in denen 
die Auswirkungen von sog. „Gebrechlichkeit" (,, frailty") aufdas Operationsrisiko und das Outcome untersucht werden. Unter Gebrechlichkeit versteht man allgemein eine Einschränkung der physiologischen Reserve und erhöhte Vulnerabilität des Patienten gegenüber auftretenden Stressfaktoren [15]. Jeder stationäre Krankenhausaufenthalt und jede Operation bedeutet solch einen Stressfaktor, im Besonderen für den alten Menschen. Leider gibt es weder eine einheitliche Definition von Frailty, ebenso wenig wie es ein einheitliches Tool gibt, um diese zu messen $[16,17]$. Anhand eines "modified frailty index" wurde für gefäßchirurgische Patienten gezeigt, dass das präoperative Screening auf Frailty eine bessere Aussage über das Mortalitätsrisiko trifft als andere etablierte Systeme zur Klassifikation des Operationsrisikos [18]. Mit einer anderen Operationalisierung von Frailty, dem sog. „clinical frailty score" (CFS), lässt sich in der Gefäßmedizin das Risiko für eine Entlassung in eine Pflegeeinrichtung und/oder eine postoperative Mobilitätseinschränkung gut vorhersagen; zudem wurde nachgewiesen, dass für gebrechliche Patienten (CFS $\geq 5$ ), ein 12fach erhöhtes Risiko einer 30-Tage-Mortalität gegenüber den als nicht gebrechlich eingestuften Patienten besteht [19].

Die alleinige Nutzung der Frailty zum präoperativen Screening ist aus geriatrischer Sicht allerdings zu kurz gefasst, werden damit doch lediglich negative Punkte gesammelt, mit denen eine Intervention oder Operation unwahrscheinlicher gemacht wird. Frailty-Scores ersetzen demnach nicht den Geriater. Mit der kompetenten Nutzung des umfassenden geriatrischen Assessments (s. oben) wird der Patient in seiner Körperfunktion und Körperstruktur, Aktivität und Partizipation sowie Umweltfaktoren charakterisiert und dadurch nicht rein Defizit-, sondern auch ressourcenorientiert klassifiziert. Damit können in der individuellen Betrachtung sehr wohl Gründe gefunden werden, die eine bestimmte (gefäß)chirurgische Operation/Intervention trotz höherem Eingriffsrisiko sinnvoll erscheinen lassen.

\section{Delir}

Das postoperativ auftretende Delir ist eine typische altersmedizinische Kom- plikation nach chirurgischen Eingriffen. In den unterschiedlichen Ausprägungen eines hyperaktiven, gemischten und eines (viel zu häufig verkannten) hypoaktiven Delirs ist dies ein Problem insbesondere beim älteren Menschen im Krankenhaus, welches ein differenziertes Management mit konsequenter Nutzung der sensorischen Hilfsmittel wie Brille und Hörgeräte, reorientierenden Maßnahmen, Wiederherstellung des Tag-Nacht-Rhythmus, Frühmobilisierung und ggf. notwendiger neuroleptischer Medikation nach sich zieht, da die in der Vergangenheit häufig gängige Praxis der Fixierungsmaßnahmen nicht nur eine maximale Verletzung der körperlichen Integrität bedeutete, sondern auch zu einer Verzögerung des Abklingens des Delirs führen konnte. Nach gefäßchirurgischen Operationen liegt die Inzidenz eines Delir zwischen 5 und 39\% (Angabe in zwei Drittel der Studien $>20 \%$ ). Dabei stellen neben dem Alter als Hauptrisikofaktor v.a. eine vorbestehende kognitive Einschränkung bzw. Depression, Bluthochdruck und offene Aortenoperationen Risikofaktoren dar [20].

Von besonderer gefäßchirurgischer Relevanz sind Amputationsoperationen und fortgesetztes Rauchen als delirauslösende Risikofaktoren [21]. Somit kann ein präoperatives Screening des Delirrisikos helfen, Hochrisikopatienten vorab zu identifizieren und diese dann mit einem perioperativ begleitenden Delirmanagement in interdisziplinärer Zusammenarbeit mit der Altersmedizin bestmöglich zu schützen. Dies kann helfen, die durch ein Delir entstehenden Komplikationen - verlängerter Aufenthalt im Krankenhaus und auf der Intensivstation, operative Komplikationen, Entlassungsnotwendigkeit in eine Heimeinrichtung und eine höhere 1-Jahres-Mortalitätsrate - zu reduzieren [21].

\section{Fallstricke für und Chancen durch einen besseren Synergismus}

In vielen operativen Bereichen im Krankenhaus ist weder ein Screening der zur elektiven Operation anstehenden alten $\mathrm{Pa}$ tienten auf das Vorliegen einer Gebrechlichkeit und schon gar nicht ein präoperatives umfassendes geriatrisches Assessment etabliert. Eine Implementierung insbesondere der geriatrischen Mitbeurteilung in das präoperative Prozedere kann möglicherweise helfen, eine bessere Entscheidung hinsichtlich einer Intervention oder Operation im Sinne der Restitutio ad optimum zu treffen. Des Weiteren kann, wenn eine Intervention notwendig, der Patient jedoch „zu frail“ erscheint, auch eine Diskussion hinsichtlich einer präoperativen Prähabilitationsmaßnahme sinnvoll sein, um die Erfolgschancen der Intervention zu verbessern. Auch die Implementierung eines Screenings des Delirrisikos mit anschließender Durchführung eines perioperativen Delirmanagements ist mit einem höheren Personalaufwand vergesellschaftet, der vielfach gescheut wird, obwohl durch eine Reduktion der Delirhäufigkeit und -intensität das Outcome des Patienten nach der Operation bzw. Intervention verbessert werden kann und auch Behandlungskosten reduziert werden könnten.

Ein Hauptgrund der unzureichenden Synergie zwischen Gefäßmedizin und Altersmedizin ist wahrscheinlich jedoch in der fehlenden Abbildung der gemeinsamen Behandlung von alten Menschen in der DRG-Vergütung (,diagnosis related groups") zu sehen. So sucht man vergeblich nach einem DRG-Code, der eine geriatrische frührehabilitative Komplexbehandlung eines Patienten mit einer gefäßmedizinischen Hauptdiagnose abbildet. Hier besteht großer Nachbesserungsbedarf bei den Krankenhaus-Budgetverhandlungen im DRG-System, sofern Gefäßmedizin und Geriatrie auch von Kostenträgerseite Hand in Hand gehen sollen.

\section{Gefäßmedizinische Entitäten im Kontext der ferneren Lebenserwartung}

Das Bauchaortenaneurysma (BAA) ist eine Erkrankung, die sich zwar schon in der vierten Lebensdekade anhand lebensstilassoziierter (Kranheits)muster mit einer gewissen Wahrscheinlichkeit erahnen lässt, allerdings wird die morphologische Manifestation aber oft erst ab der siebten Lebensdekade sichtbar; von da an steigt die Prävalenz stetig bis in die zehnte Lebensdekade an. Der Manifestationsverlauf eines BAA geht also einher mit der Großlebensphase des Alters inklusive kalendarischer Zwischenstufungen der "young-olds“ (65-74 Jahre), 
„middle-olds" (75-84 Jahre) und „old(est)olds" ( $\geq 85+$ Jahre; [22]) und kann insofern als Paradigma für Perspektiven, aber auch Grenzen, eines modernen Ko-Managements zwischen Gefäßchirurgie und Geriatrie gelten. In diesem Sinne ist das BAA eine regelhaft anzutreffende geriatrische Begleiterkrankung, wobei sich der Begriff geriatrisch nicht so sehr auf das Lebensalter $\geq 70$ respektive $\geq 80$ Jahre, sondern definitionsgemäß die in diesem Alter interindividuell unterschiedlich anzutreffende Multimorbidität respektive Vulnerabilität unterstreicht. Dabei sind geriatrietypische Multimorbidität und Vulnerabilität schon im klinischen Alltag eng ausgelegte Fachbegriffe, die ebenso wie der schon oben eingeführte Begriff der Frailty differenziert und in definitorischer Übereinkunft verwendet werden sollten $[9,23]$.

Ein konsequent umgesetztes BAAScreening bei allen Menschen ab 70 Jahren würde zu einer Detektion im GröBenbereich von 1:15 beim Mann und 1:40 bei der Frau führen [24]. Bei dann strikter Einleitung weiterer Verlaufskontrollen und Behandlungsmaßnahmen kämen die zur Verfügung stehenden gefäßmedizinischen Ressourcen rasch an ihre Grenzen. Allerdings bedeutet ein (zu) früh gefälltes Behandlungsurteil für oder gegen eine engmaschige Beobachtung mit (schlussendlicher) endovaskulärer/ offener Behandlung auch eine definitive Entscheidung für oder gegen den aktiven Versuch der Verhinderung einer potenziell eintretenden tödlichen Ruptur des BAA.

Tatsächlich wurden Überlegungen, ob und inwieweit die individuelle Gefahr einer tödlichen Ruptur bei diagnostiziertem BAA mit der durch das kalendarische Alter vorgegebenen, demografischen Überlebenswahrscheinlichkeit abgeglichen werden sollte, aus aktuellem Anlass angestellt [25]: So gab das American College of Surgeons vor dem Hintergrund der SARSCoV-2-Pandemie („,severe acute respiratory syndrome coronavirus 2") die Empfehlung aus, möglichst jegliche Revision von BAA - auch bei einem Durchmesser $>6,5 \mathrm{~cm}$ auf einen späteren Zeitpunkt zu verlegen [26].

Derartige statistische Modellrechnungen anhand des kalendarischen Alters und der damit verbundenen sog. fer- neren Lebenserwartung können epidemiologisch-methodisch (vgl. zugrunde gelegte Periodensterbetafeln) allenfalls eine grobe Orientierung darstellen und vor dem Hintergrund der im Alter besonders heterogenen, intervenierenden Faktoren nur einer von vielen Gesichtspunkten bei der individuellen medizinischen Entscheidungsfindung sein. Wie kurz eine epidemiologisch gestützte Fokussierung allein auf das kalendarische Alter greift, kann man - pandemieunabhängig - am jährlichen Rupturrisiko von BAA darlegen, das in den AWMF-Leitlinien (Arbeitsgemeinschaft Wissenschaftlicher Medizinischer Fachgesellschaften) der DGG von 2018 als gepooltes Risiko ab einem Durchmesser von $\geq 5,5 \mathrm{~cm}$ mit $4 \%$ aufwärts angegeben wird [24]. Gegenüber den aktuellen Periodensterbetafelbasierten Daten zum durchschnittlichen demographischen 1-Jahres-Überleben in Deutschland wären diese „zusätzlichen“ $4 \%$ Rupturgefahr bei einem 65-Jährigen mit 98\% 1-Jahres-Überleben und auch bei einem 75-Jährigen mit $97 \%$ (1-JahresÜberleben) beträchtlich; bei einem 85Jährigen mit einer 1-Jahres-Überleben von $90 \%$ erschienen sie etwas hinnehmbarer. Nichts desto weniger ergibt sich - auch und gerade beim 85-jährigen BAA-Patienten - die Notwendigkeit, den Behandlungsplan zusätzlich und personalisiert am individuellen funktionalen Gesundheitsstatus festzumachen. Funktionale Gesundheit beschreibt dabei (a.) defizitorientiert (pathogenetisch) die Interaktion von multiplen Beeinträchtigungen der Körperfunktion und Körperstruktur und ist (b.) umgekehrt ressourcenorientiert (salutogenetisch) Ausdruck der bis dahin erhaltenen oder verloren gegangen Ressourcen. Surrogatparameter für funktionale Gesundheit, wie es z. B. Scores für Multimorbidität und Frailty implizieren, werden diesem komplexen Gefüge nicht gerecht, weshalb es gerade bei so bedeutenden elektiven Entscheidungen wie der Therapie eines BAA beim alten Menschen Sinn macht, ein fachmedizinisches geriatrisches Assessment einzuholen [11, $12]$.
Durch die richtungsweisende getABIStudie $^{2}$ aus den 2000er-Jahren [27] wissen wir, dass in Deutschland jede(r) Fünfte über 65 Jahre einen Knöchel-Arm-Index (ABI) von $<0,9$ aufweist und damit die notwendige Diagnosevoraussetzung für eine periphere arterielle Verschlusskrankheit (pAVK) erfüllt. Mit ihrer Altersbeschränkung von $\geq 65$ Jahren deckt sich die getABiStudie dem sozialwissenschaftlich-gerontologischen Eintrittswert für die Großlebensphase des Alterns, das Durchschnittalter der getABI-Studie von 72,5 Jahren resultiert jedoch aus den $>90 \%$ Studienteilnehmern, die entweder "young-olds" (65-74 Jahre) oder allenfalls als „middleolds" (75-84 Jahre) waren [22]. Abgeglichen mit aktuellen demographischen Daten zur ferneren Lebenserwartung in Deutschland [28] ist die entsprechend getABI-Studie mit pAVK einhergehende angegebene 5-Jahres-Sterblichkeit im Bereich von $20 \%$ [29] für eine 72 -Jährige mit einer sterbetafelbasierten 5-JahresÜberlebenswahrscheinlichkeit von $91 \%$ beachtlich, für einen 72-Jährigen jedoch angesichts seiner demographischen 5-Jahres-Überlebenswahrscheinlichkeit von bereits $85 \%$ verhältnismäßig unspektakulär.

Eine komplementäre epidemiologische Studie zur neu diagnostizierten pAVK bei Männern erbrachte unter Einbezug der Hochaltrigkeit eine genauere Differenzierung in der Mortalität: Demnach verstarben rund $6 \%$ der sog. „youngolds" (65-74 Jahre), 9\% der "middle-old" (75-84 Jahre) und $15 \%$ der "old(est)-old" (85+ Jahre) im Zeitraum von einem Jahr nach der Erstdiagnose einer pAVK [30]. Wiederum abgeglichen mit der aktuellen sterbetafelbasierten Daten zur 1-JahresÜberlebenswahrscheinlichkeit in Deutschland [28] wäre dies bei einem 65-Jährigen (98\%) und auch bei einem 75-Jährigen (97\%) immens, bei einem 85-Jährigen angesichts seiner 1-Jahres-Überlebenswahrscheinlichkeit von $90 \%$ jedoch eher verhältnismäßig.

2 German Epidemiological Trial on Ankle Brachial Index. 


\section{Fazit für die Praxis}

- Trotz der fachlich zahlreichen Überschneidungen gibt es in der Praxis bisher nur wenig gelebte Kooperationen zwischen den Fachdisziplinen der Alters- und der Gefäßmedizin. Hier besteht ein Optimierungsbedarfs sowohl auf der Ebene der Krankenversorgung als auch bei wissenschaftlichen Studien.

- Bisher häufig herangezogene Surrogatparameter wie das kalendarische Alter oder Frailty reichen nicht aus, um den komplexen geriatrischen Gefäßpatienten ausreichend individuell zu erfassen.

- Daher ist komplementär zur gefäßmedizinischen Fachexpertise ein umfassendes geriatrisches Assessment wünschenswert, um sich auch bei hochaltrigen Patienten individuell besser für oder gegen eine interventionelle oder operative Therapie entscheiden zu können.

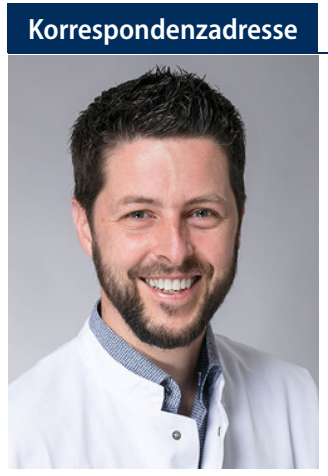

\section{Björn Maassen}

Medizinische Klinik VI (Altersmedizin), Uniklinik RWTH Aachen

Pauwelsstraße 30, 52074 Aachen, Deutschland bmaassen@ukaachen.de

Förderung. Der Aufbau des Lehrstuhls für Altersmedizin der Uniklinik RWTH Aachen wird unterstützt von der Robert Bosch Stiftung (32.5.1140.0009.0).

\section{Einhaltung ethischer Richtlinien}

Interessenkonflikt. B. Maassen, K. Chondros und L.C. Bollheimer geben an, dass kein Interessenkonflikt besteht.

Für diesen Beitrag wurden von den Autoren keine Studien an Menschen oder Tieren durchgeführt. Für die aufgeführten Studien gelten die jeweils dort angegebenen ethischen Richtlinien.

\section{Literatur}

1. Rapp K, Becker C, Todd C, Rothenbacher D, Schulz C, König HH, Liener U, Hartwig E, Büchele G (2020) Assoziation von orthogeriatrischem CoManagement und Sterblichkeit nach Hüftfraktur.

\section{Innovative geriatric concepts for vascular medicine and vascular surgery}

So far, advances in vascular surgery and geriatrics have developed largely independent of each other, and there are - unlike orthogeriatrics - hardly any overlaps in daily clinical practice. Yet in an interdisciplinary setting, geriatric concepts might be helpful in individualized indication, choice of therapy and prognosis in vascular surgery, especially in terms of old(est)-old patients (85+). Geriatric notions, such as biological age and functionality, are not sufficiently reflected by the vascular status, but have to consider other organ-specific components (e.g., immunology, musculoskeletal system) as well as psychological/neurocognitive issues and social contextual factors. Limitations to chronological age, the vascular status or paraphrasing geriatric surrogate parameters like "frailty" do not properly characterize the functional health status of old people. In the present article we follow this train of thought at the interface between vascular surgery and geriatrics using the examples of abdominal aortic aneurysm (AAA) and peripheral arterial occlusive disease (PAOD).

\section{Keywords}

Abdominal aortic aneurysm · Periphere arterial occlusive disease - Multimorbidity · Polypharmacy . Frailty
Eine Beobachtungsstudie mit 58000 Patienten aus 828 Krankenhäuser. Dtsch Arztebl Int 117:53

2. Halandras PM (2019) Vascular surgery and geriatric patients. Clin Geriatr Med 35:93

3. Kaschwich $M$, von Kodolitsch $Y$, Tsilimparis $N$, Behrendt CA, Sachweh A, Debus ES (2017) Gefäßchirurgie bei alten Menschen: Was ist möglich? Was ist sinnvoll? Chirurg 88:116

4. Böckler D, Schumacher H, Allenberg JR (2005) Gefässchirurgie im Alter. Chirurg 76:113

5. DIMDI (2005) ICF-Modell. http: //www.dimdi.de/ dynamic/de/klassi/downloadcenter/icf.Zugegriffen: 9. Apr. 2021

6. Chase P, Mitchell K, Morley JE (2000) In the steps of giants: the early geriatrics texts. J Am Geriatr Soc 48:89

7. Masnoon N, Shakib S, Kalisch-Ellett L, Caughey GE (2017) What is polypharmacy? A systematic review of definitions. BMC Geriatr 17:230

8. Johnston MC, Crilly M, Black C, Prescott GJ, Mercer SW (2019) Defining and measuring multimorbidity: a systematic review of systematic reviews. Eur JPublic Health 29:182

9. DGG, DGGG, BVG (2007) Definition des geriatrischen Patienten. https://www.bv-geriatrie.de/ images/pdf_word_dateien/politik_recht/BVG_ Broschuere_Bundesweites_Geriatriekonzept_ Web.pdf.Zugegriffen: 15. Apr. 2021

10. Parker SG, McCue P, Phelps K, McCleod A, Arora S, Nockels K, Kennedy S, Roberts H, Conroy S (2018) What is comprehensive geriatric assessment (CGA)? An umbrella review. Age Ageing 47:149

11. DEGAM et al (2018) Geriatrisches Assessment in der Hausarztpraxis (AWMF 053-15). https:// www.awmf.org/leitlinien/detail/ll/053-015.html. Zugegriffen:22. Apr. 2021

12. DGG et al (2019) Geriatrisches Assessment der Stufe (AWMF 84-002). https://www.awmf.org/ uploads/tx_szleitlinien/084-002l_GeriatrischesAssessment-Stufe-2_2019-08_01.pdf.Zugegriffen:25. Apr. 2021

13. Pazan F, Weiss C, Wehling M (2019) The FORTA (fit fOR the aged) list 2018: third version of a validated clinical tool for improved drug treatment in older people. Drugs Aging 36:481
14. Holt S, Schmiedl S, Thürmann PA (2011) PRISCUSListe potenziell inadäquater Medikation für ältere Menschen. https://media.gelbe-liste.de/ documents/priscus-liste.pdf. Zugegriffen: 6. Mai 2021

15. Jacobs AH, Bollheimer LC (2019) Frailty. In: Maetzler W, Dodel R, Jacobs AH (Hrsg) Neurogeriatrie. Springer, Berlin Heidelberg, $\mathrm{S} 49$

16. Hoogendijk EO, Afilalo J, Ensrud KE, Kowal $P$, Onder G, Fried LP (2019) Frailty: Implications for clinical practice and publichealth. Lancet 394:1365

17. Mende A, Riegel AK, Plümer L, Olotu C, Goetz AE, Kiefmann R (2019) Gebrechliche ältere Patienten Determinanten des perioperativen Verlaufs. Dtsch Arztebl Int 116:73

18. Ehlert BA, Najafian A, Orion KC, Malas MB, Black JH 3rd, Abularrage CJ (2016) Validation of a modified Frailty Index to predict mortality in vascular surgery patients. JVasc Surg 63:1595

19. Donald GW, Ghaffarian AA, Isaac F, Kraiss LW, Griffin CL, Smith BK, Sarfati MR, Beckstrom JL, Brooke BS (2018) Preoperative frailty assessment predicts loss of independence after vascular surgery. JVasc Surg 68:1382

20. Aitken SJ, Blyth FM, Naganathan V (2017) Incidence, prognostic factors and impact of postoperative delirium after major vascular surgery: a meta-analysis and systematic review. Vasc Med 22:387

21. Visser $L$, Prent $A$, van der Laan $M J$, van Leeuwen $B L$, Izaks GJ, Zeebregts CJ, Pol RA (2018) Predicting postoperative delirium after vascular surgical procedures. JVasc Surg 62:183

22. Cohen-Mansfield J, Shmotkin D, Blumstein Z, Shorek A, Eyal N, Hazan H, CALAS Team (2013) The old, old-old, and the oldest old: continuation or distinct categories? An examination of the relationship between age and changes in health, function, and wellbeing. Int J Aging Hum Dev 77:37

23. MDS (2018) Begutachtungsanleitung Vorsorge und Rehabilitation, Seite $39 f$ u S77. https://www.mds-ev.de/fileadmin/dokumente/ Publikationen/GKV/Begutachtungsgrundlagen GKV/BGA_Vorsorge-Reha_18-07-02.pdf. Zugegriffen: 15. Apr. 2021 
24. DGG (2018) S3-Leitlinie: Screening, Diagnostik, Therapie und Nachsorge des Bauchaortenaneurysmas (AWMF 004-014). S10, S14 u S19. https:// www.awmf.org/leitlinien/detail/ll/004-014.html. Zugegriffen: 15.Apr. 2021

25. McGuinness B, Troncone M, James LP, Bisch SP, lyer V (2021) Reassessing the operative threshold for abdominal aortic aneurysm repair in the context of COVID-19. JVasc Surg 73:780

26. ACS (2020) COVID-19 guidelines for triage of vascular surgery patients. https://www.facs. org/covid-19/clinical-guidance/elective-case/ vascular-surgery.Zugegriffen: 15. Apr. 2021

27. Diehm C, Allenberg JR, Pittrow D, Mahn M, Tepohl G, Haberl RL, Darius H, Burghaus I, Trampisch HJ (2009) German epidemiological trial on ankle brachial index study group. Mortality and vascular morbidity in older adults with asymptomatic versus symptomatic peripheral artery disease. Circulation 120(21):2053-2061

28. Destatis (2020) https://www.destatis.de/DE/ Themen/Gesellschaft-Umwelt/Bevoelkerung/ Sterbefaelle-Lebenserwartung/Publikationen/ Downloads-Sterbefaelle/periodensterbetafelerlaeuterung-5126203197004.pdf? blob=publicationFile. Zugegriffen: 10 . Apr. 2021

29. Lawall H, Diehm C, Hoffmann U, Reinecke H (2015) Periphere arterielle Verschlusskrankheit: Epidemiologie, Komorbidität und Prognose [Update PAVK: Epidemiology, comorbidity and prognosis of peripheral arterial obstructive disease. Dtsch Med Wochenschr 140(24):1798-1802

30. Willey J, Mentias A, Vaughan-Sarrazin M, McCoy K, Rosenthal G, Girotra S (2018) Epidemiology of lower extremity peripheral artery disease in veterans. J Vasc Surg 68(2):527-535

\section{Christoph Klein, Jan-Maximilian Zeller Strafrechtliche Risiken des Arztes}

\section{Ecomed Medizin Verlag 2021, 168 S., (ISBN: 978-3-609-16538-7), 39,99 EUR}

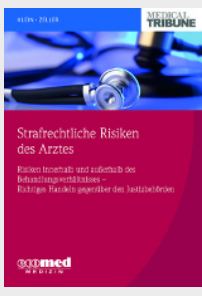

Fachgebiet beherrschen, sondern auch sich für die generellen, juristischen Rahmenbedingungen interessieren.

Das Buch Strafrechtliche Risiken des Arztes beleuchtet in erfrischend verständlicher Sprache prägnant die juristischen Aspekte im klinischen Alltag.

Das Buch ist in drei Sektionen unterteilt. Der erste Abschnitt bezieht sich konkret auf Handlungsabläufe in der Patientenversorgung. Es werden die im Alltag relevanten medizinischen Teilbereiche abgehandelt. Beginnend mit der Thematik "Aufklärung und Einwilligung", über urologische Tätigkeitsschwerpunkte, allgemeine, ärztliche Hilfspflichten, Aspekte bezüglich Zeugnisausstellungen, Schweigepflicht oder auch Sterbehilfe werden die jeweiligen Kapitel für den Kliniker mit Verweis auf die entsprechenden Gesetze fokussiert vorgestellt.

Der zweite Abschnitt behandelt das Themenfeld außerhalb der Behandlung im Praxisalltag. Unter anderem werden die Tatbestände Korruption, strafbare Werbung und Abrechnungsbetrug beleuchtet.

Der letzte Abschnitt behandelt das Auftreten gegenüber Justizbehörden. Dieser Abschnitt ist ebenfalls ein wesentlicher Bestandteil, da nicht nur Verhaltensregeln in der Rolle des Beschuldigten, sondern auch bei Durchsuchungen dargelegt werden. Auch die standesrechtlichen Aspekte bzw. Konsequenzen werden erläutert.
Die Kapitel sind verständlich aufgebaut und wichtige Informationen werden farblich prägnant hervorgehoben. Fallbeispiele unterstützen die Vermittlung der Inhalte didaktisch sinnvoll.

Dieses Buch ist für klinisch tätige Ärzte absolut lesenswert und nützlich, um die Aufmerksamkeit für juristische bzw. strafrechtliche Gegebenheiten zu schärfen. 